\title{
ВИРТУАЛЬНАЯ РЕАЛЬНОСТЬ \\ (VIRTUAL REALITY)
}

\author{
Информатика
}

\section{Моросеев Д. А.}

2. Тверь, Тверской лищей, 10 класс

Научный руководитель: Наумова А.И., г. Тверь, преподаватель информатики высшей категории, Тверской лищей

Данная статья является реферативным изложением основной работы. Полный текст научной работы, приложения, иллюстрации и иные дополнительные материалы доступны на сайте IX Международного конкурса научно-исследовательских и творческих работ учащихся “Старт в науке” по ссылке: https://school-science.ru/9/4/44359.

В данной работе представлен материал о виртуальной реальности (Virtual Reality). Тема достаточно актуальная и представляет повышенный интерес для учащихся профильных классов.

Цель данной работы заключается в том, чтобы получить дополнительные знания по этой теме и практические навыки по разработке виртуальной модели на примере создания компьютерной модели полусумматора в программной среде языка объектно-ориентированного программирования Delphi.

Задача состоит в том, чтобы подобрать соответствующий материал с последующей систематизацией, обобщением и иллюстрацией текста.

Работа состоит из двух частей: теоретической (даны определения, свойства, типы, оборудование, области применения VR) и практической (приведён пример разработки инженерного проекта: формальное описание алгоритма с использованием логических выражений, создание программного кода с комментариями на русском языке и последующей компиляцией его в выполняемый файл, подготовка тестовых примеров, проведение компьютерного эксперимента и анализ полученных результатов. 


\section{Виртуальная реальность (Virtual Reality)}

\section{Определение VR}

VR (виртуальная реальность) - это искусственно созданный трехмерный uңифровой мир, позволяющий переместить человека в любое время и место. С помощью различных сенсоров и периферийных устройств виртуальный мир ощущается почти как реальный.

$\mathrm{AR}$ (дополненная реальность) - это технология добавления в поле восприятия человека виртуальной информации, которая воспринимается как элементы реальной жизни.

Таким образом, если в виртуальной реальности человек находится в полностью цифровом окружении, имитирующем реальный мир, то в дополненной реальности пользователь остается в окружающем пространстве, но его опыт расширяется путем добавления виртуальных компонентов. [7]

\section{История создания}

1956 год. Sensorama - 3D-дисплеи: На первый взгляд устройство походило на какое-то медицинское оборудование, но на самом деле это была одна из первых попыток отгородиться от реального мира. Sensorama имела стереозвук, 3D-дисплей, небольшой генератор и даже посадочное место. Это было кинематографическое детище Morton Heilig, который подготовил шесть короткометражных фильмов (рис. 1). [5]

1961 год. Headsight - начальник слежения: Инженеры корпорации Phlico свели в одном шлеме видеоэкран с базовой системой отслеживания и связали все для CCTV. Основная цель Headsigh состояла в том, чтобы удаленно наблюдать ситуации, которые слишком опасны, чтобы находиться в непосредственной близости от них (рuс. 1). [5]

1966 год. GAF Viewmaster-стереоскопический 3D: Знаковый красный стереоскоп объединял несколько отличающихся друг от друга изображения одной и той же сцены, позволяющих создать единое 3D-изображение. Устройство первым дало теперь уже знакомое каждому ощущение погружения в другой мир (рuс. 1). [5] 
1968 год. Sword of Damocles-AR графика: Также известно как система передвижного дисплея (HMD). Это был первый опыт Линкольновской лаборатории Массачусетского технологического института, и предполагала наложение геометрической сетки на зрение пользователя. Это была первая система, способная заменить камеру с компьютером. Сложность заключалось в том, что все составные части системы были настолько тяжелыми, что устройство должно было быть подвешено к потолку (рuс. 1). [5]

1980 год. Еуе Тар-миниатюризация: Стив Манн создал громоздкий рюкзак-компьютер, подключенный к шлем-камере и видоискателю. Манн запряг расщепитель луча, чтобы демонстрировать сцену, как пользователю, так и компьютеру, подключенному к камере, что позволяло производить наложение данных в реальном времени (рис. 1). [5]

1984 год. RB2-контролеры First VR: RB2 была первой коммерческой системой VR и включала в себя перчатки, позволяющие пользователям крутить и переворачивать виртуальные объекты, которые появлялись на дисплее EyePhone (puc. 1). [5]

1985 год. NASA - ЖК-оптика и головка слежения: На протяжении последних четырех десятилетий Космическое агентство играло важную роль в разработке VR, комбинируя светодиоды, жидкокристаллические дисплеи и широкоугольную оптику, позволяющие создать хороший эффект виртуальной реальности. Тогда же, в 80-е впервые появились технологии отслеживания (puc. 1). [5]

1995 год. CAVE-Несколько пользователей: В CAVE (автоматическая виртуальная среда) использовались стереоскопические ЖК-затворные очки и настенные проекции, позволяющие создавать трехмерную сцену, через которую пользователь мог бы пройти. Технология была разработана студентами Университета штата Иллинойс. Им удалось создать легкие VR-очки, больше похоже на те, что производят сегодня. Новаторство CAVE заключалось еще и в том, что в виртуальном пространстве могли находиться одновременно несколько пользователей (рис. 1). [5] 


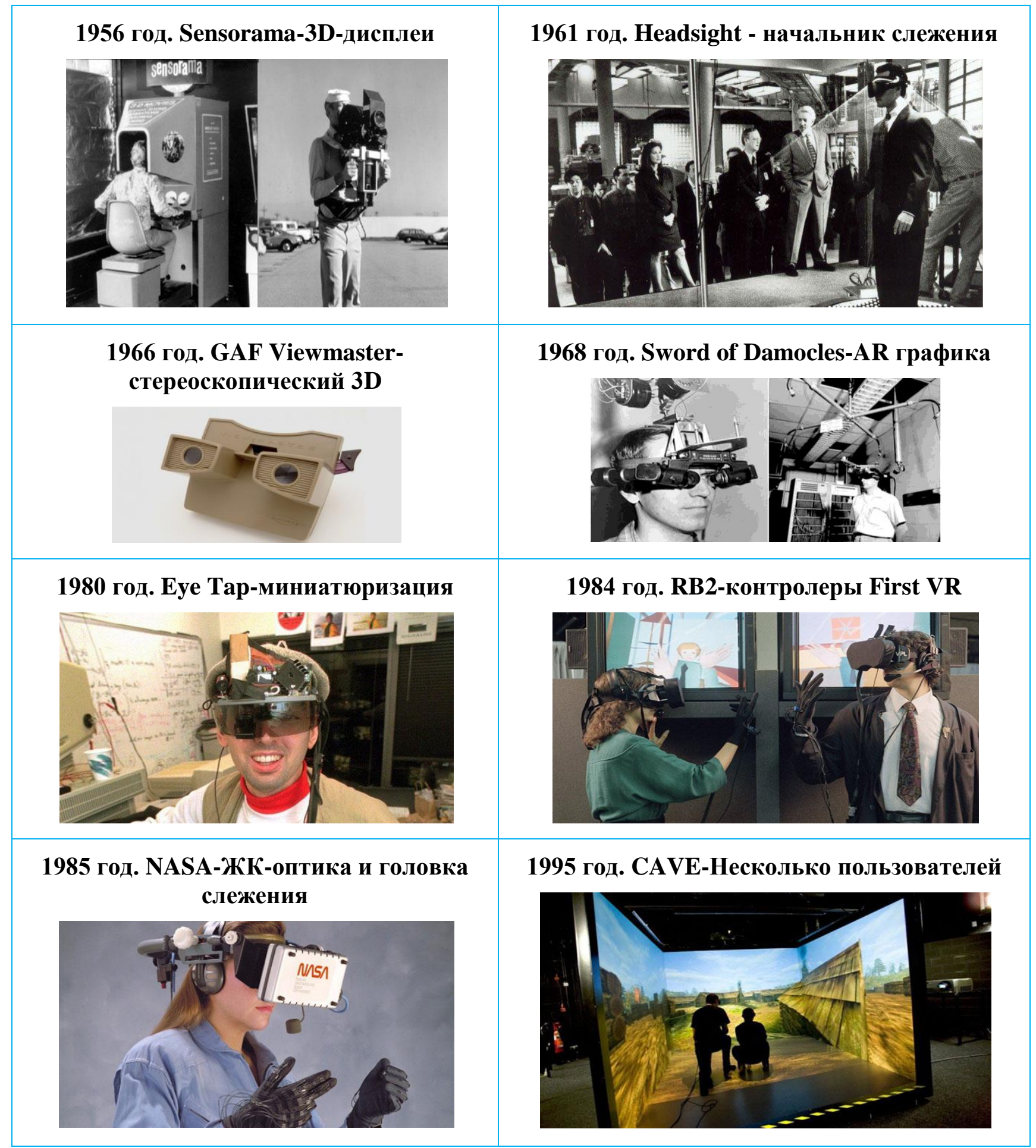

Рис. 1. История создания VR

\section{Свойства VR}

Полный набор встретить можно редко, но ниже перечислены те особенности, на которые нужно ориентироваться при создании виртуальной реальности: Правдоподобная - поддерживает у пользователя ощущение реальности происходящего; Интерактивная - обеспечивает взаимодействие 
со средой; Машинно-генерируемая - базируется на мощном аппаратном обеспечении; Доступная для изучения - предоставляет возможность исследовать большой детализированный мир; Создающцая эффект присутствия - вовлекает в процесс как мозг, так и тело пользователя, воздействуя на максимально возможное число органов чувств. [8]

\section{Tипы VR}

VR с эффектом полного погружения: Этот тип подразумевает наличие

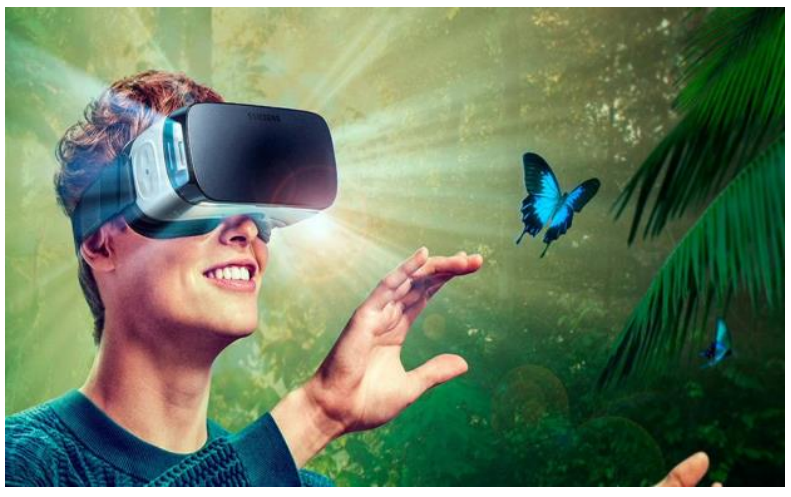
трёх факторов:

1. Правдоподобная симуляция мира с высокой степенью детализации.

2.Высокопроизводительный компьютер, способный распознавать действия пользователя и реагировать на них в

режиме реального времени.

3.Специальное оборудование, соединенное с компьютером, которое обеспечивает эффект погружения в процессе исследования среды. [8]

VR без погружения: К типу “без погружения" относятся симуляции с качественным изображением, звуком и контроллерами, в идеале транслируемые на широкоформатный экран. Также в эту категорию попадают такие проекты, как археологические 3D-реконструкции древних поселений или модели зданий, которые архитекторы создают для демонстрации своей работы клиенту. [8]

VR с совместной инфраструктурой: Сюда можно отнести “виртуальные

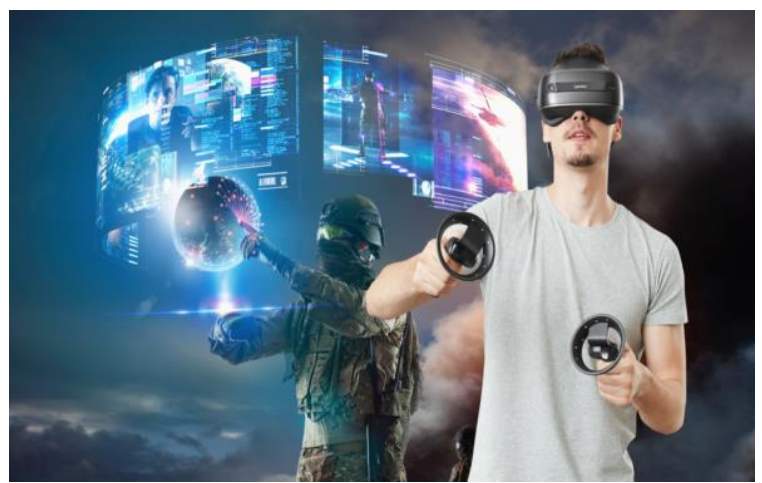
миры" вроде Second Life и Minecraft. Виртуальные миры используются не только в игровой индустрии: благодаря таким платформам, как $\underline{\text { 3D Immersive }}$ Collaboration и Open Cobalt можно организовывать рабочие и учебные $3 D$ - 
пространства - это называется “совместная работа с эффектом присутствия".

Создание возможности одновременного взаимодействия в сообществе и полного погружения сейчас является одним из важных направлений развития. [8]

VR на базе интернет-технологий: Специалисты в области компьютерных наук разработали способ создания виртуальных миров в Интернете, используя технологию Virtual Reality Markup Language, аналогичную HTML. [8]

\section{Оборудование VR}

Шлемы и очки: Такие устройства состоят из двух небольших экранов, расположенных напротив каждого глаза, штор, предотвращающих попадание внешнего света, и стереонаушников. Экраны показывают слегка смещенные друг относительно друга стереоскопические изображения, обеспечивая реалистичное $3 D$-восприятие.

В шлемах также содержатся встроенные акселерометры и датчики положения (рис. 2). [8]

VR - комната: Изображения в данном случае транслируются не в шлем, а на стены помещения, часто представляющие собой дисплеи MotionParallax3D (хотя для более полного UX в некоторых таких комнатах нужно надевать 3Dочки или даже комбинировать CAVE и HMD). Есть мнение, что VR-комнаты гораздо лучше VR-шлемов: более высокое разрешение и самоидентификация происходит проще благодаря тому, что пользователь имеет возможность постоянно себя видеть (рис. 2). [8]

Информационные перчатки VR: Для удовлетворения инстинктивной потребности пользователя потрогать руками то, что он находит для себя интересным в процессе изучения среды, были созданы перчатки с сенсорами для захвата движений кистей и пальцев рук. Техническое обеспечение такого процесса варьируется - возможно использование оптоволоконных кабелей, тензометрических или пьезоэлектрических датчиков, a также электромеханических приспособлений (рис. 2). [8] 
Джойстики (геймпады): Специальные устройства для взаимодействия с виртуальной средой, содержащие встроенные датчики положения и движения, а также кнопки и колеса прокрутки, как у мыши. Сейчас их всё чаще делают беспроводными, чтобы избежать неудобств и нагромождений при подсоединении к компьютеру (рис. 2). [8]

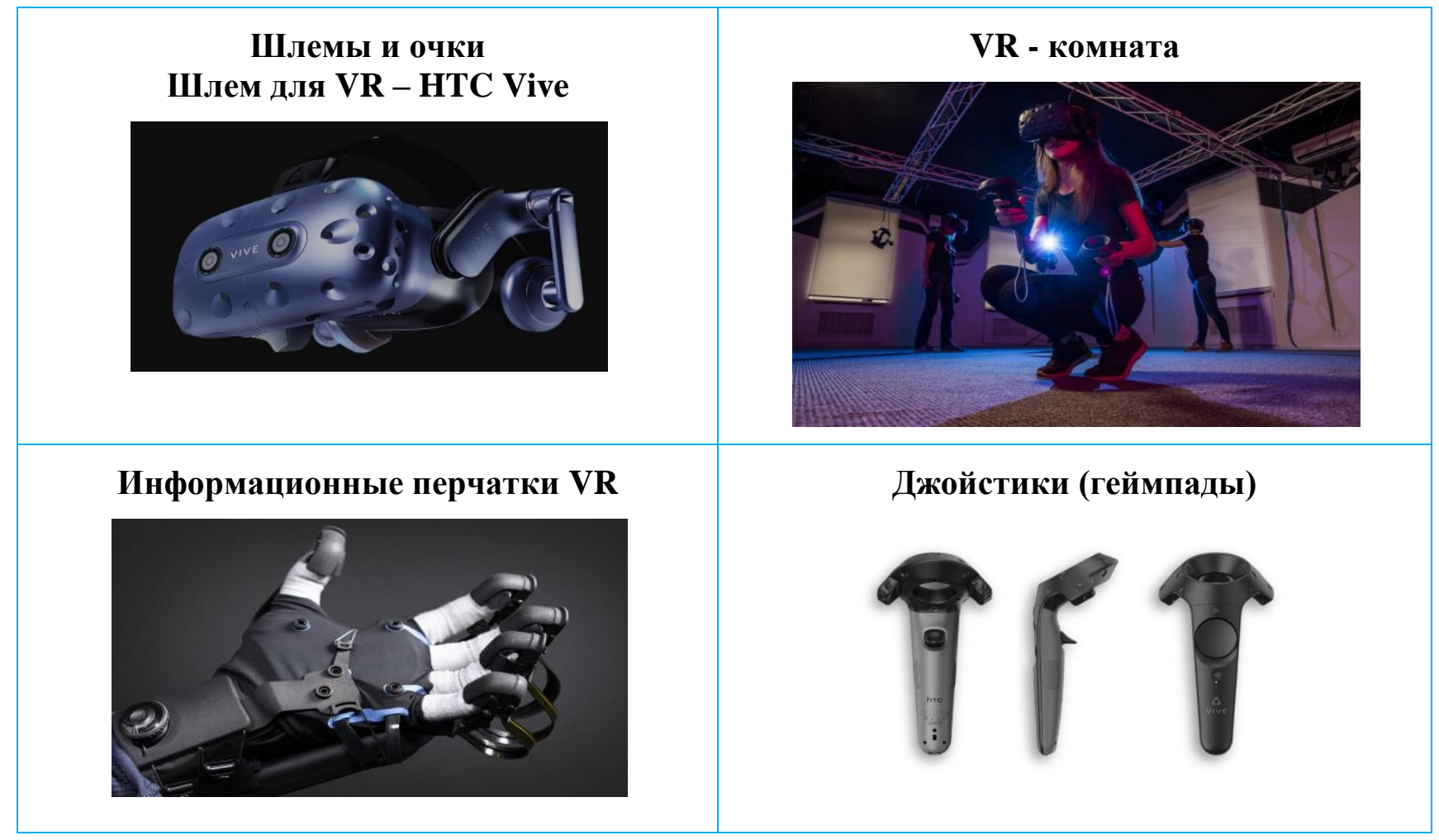

Рис. 2. Оборудование VR

\section{Области применения VR}

Обучение: VR используется для моделирования среды тренировок в тех занятиях, в которых необходима предварительная подготовка: например, управление самолетом, прыжки с парашютом и т.д. (рис. 3). [8]

$\mathrm{C}$ применением технологий VR и AR учащиеся средних школ и студенты высших учебных заведений смогут взаимодействовать с предметами в виртуальном пространстве или участвовать в важных исторических событиях (puc. 3). [1]

Медицина: Технология VR оказывается полезной на операциях: врач, используя специальное оборудование, может управлять движениями робота, получая при этом возможность лучше контролировать процесс. 
B российской медицине VR достаточно успешно применяются на этапе реабилитаџии паџиентов. [8]

В 2020 году в России запущено 16 проектов - от искусственного интеллекта до сверхчувствительных квантовых сенсоров (рис. 3). [2]

Наука: VR позволяет улучшить и ускорить исследование молекулярного и атомного мира: погружаясь в виртуальную среду, ученый может обращаться с частицами так, будто это кубики LEGO (рис. 3). [8]

Культура и искусство: Эксперименты в сфере изобразительного искусства при помощи VR/AR-технологий имеют очень широкий спектр. Наиболее распространено создание VR-картин с помощью специальных гаджетов. Не менее популярно среди художников добавление собственных элементов в существующие полотна. Также распространен опыт воссоздания музейных экспонатов в VR (рис. 3). [6]

Проектирование: Вместо того, чтобы строить дорогостоящие модели машин, самолетов или зданий, можно создать виртуальную модель, позволяющую не только исследовать проект изнутри, но и проводить тестирование его технических характеристик (рис. 3). [8]

Военная промышленность: Новейшие технологии применяются в обучении летчиков, пехоты, военных медиков и позволяют солдатам побывать в условиях, максимально приближённых к боевым, без всякой опасности для жизни и здоровья.

Дополненная реальность помогает проводить планирование военных операций. Начиная от разбора задач с использованием виртуальных моделей, до отработки конкретных военных действий (рис. 3). [1, 3]

Игры и развлечения: На данный момент это самая известная и самая широкая область использования VR: сюда входят как игры, так и кино, виртуальный туризм и посещение различных мероприятий (рис. 3). [8]

VR продолжает интегрироваться с разными сферами нашей жизни и из мифа научной фантастики она превратилась в (виртуальную) реальность. [8] 


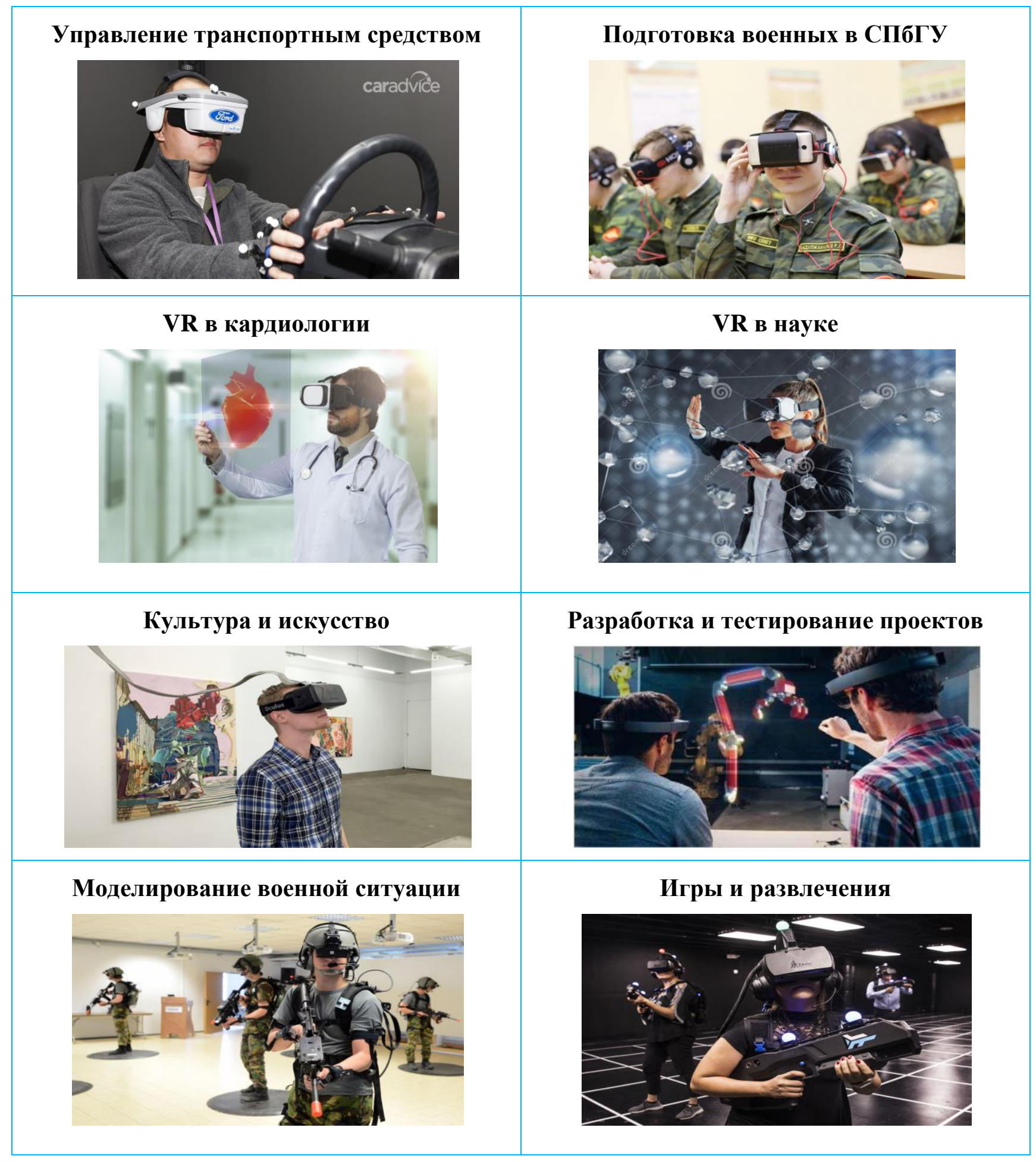

Рис. 3. Области применения VR

Проектирование инженерных разработок

Разработка компьютерной модели полусумматора на языке Delphi Формальная модель проекта

При изучении логических устройств компьютера (сумматор, триггер) целесообразно использовать компьютерные модели. Такие модели позволяют 
визуализировать прочесс преобразования логических значений входных сигналов в значения выходных сигналов.

В целях максимального упрощения работы компьютера всё многообразие математических операций в процессоре сводится к сложению двоичныхх чисел.

Для разработки проекта необходимо: построить таблищу истинности (табл. 1); на основе полученных логических выражений из базовых логических элементов можно построить схему полусумматора (рис. 4). [4]

Таблиц̧а 1. Таблица истинности

\begin{tabular}{|c|c|c|c|c|c|}
\hline $\mathbf{A}$ & $\mathbf{B}$ & $\mathbf{A} \mathbf{V} \mathbf{B}$ & $\mathbf{A} \& \mathbf{B}$ & $\neg(\mathbf{A} \& \mathbf{B})$ & $(\mathbf{A} \mathbf{V} \mathbf{B}) \boldsymbol{\&} \neg(\mathbf{A} \& \mathbf{B})$ \\
\hline 0 & 0 & 0 & 0 & 1 & 0 \\
\hline 0 & 1 & 1 & 0 & 1 & 1 \\
\hline 1 & 0 & 1 & 0 & 1 & 0 \\
\hline 1 & 1 & 1 & 1 & 0 & 1 \\
\hline
\end{tabular}

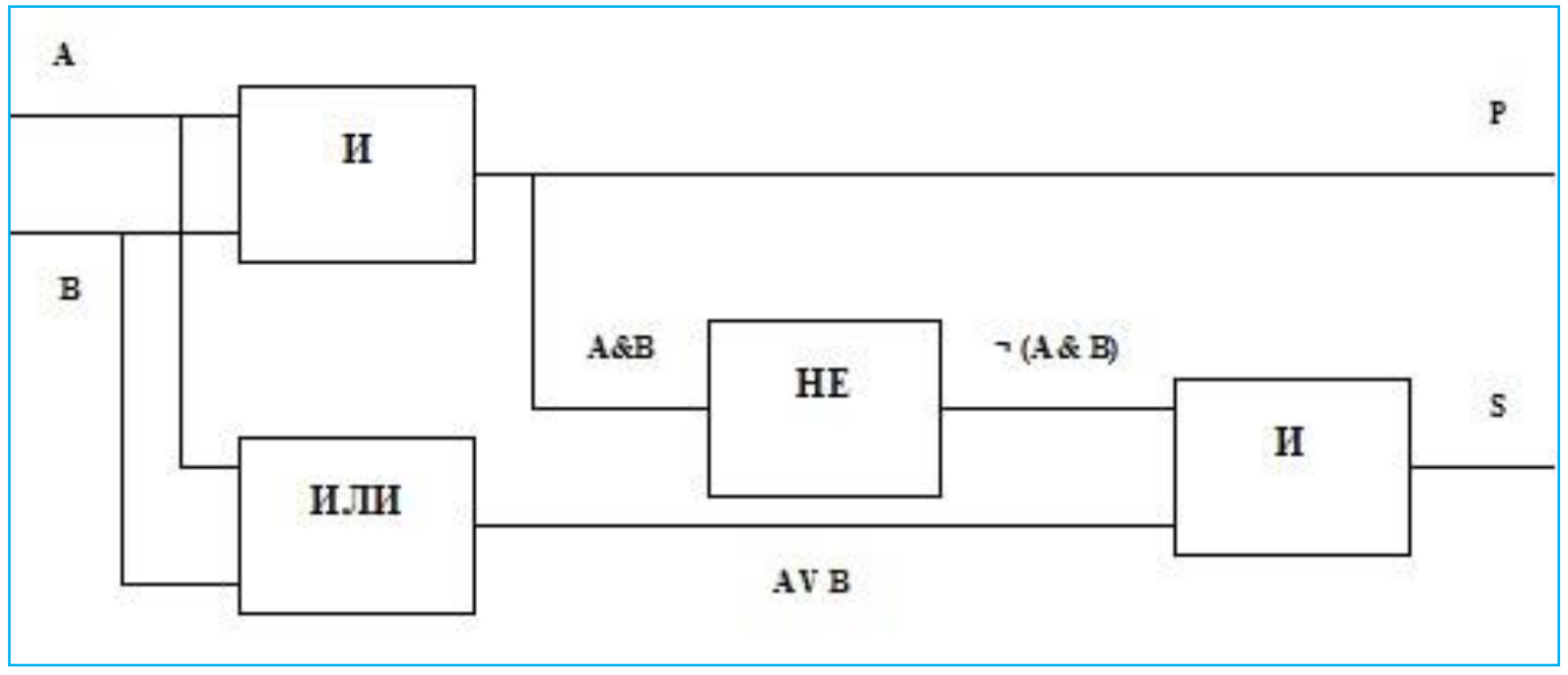

Рис. 4. Полусумматор двоичных чисел

\section{Компьютерная модель проекта}

Разработка интерфейса: поместить на форму командную кнопку Button1, 4 метки Label для изображения базовых логических элементов, 2 текстовых поля Edit для ввода логических значений на входе полусумматора, 4 метки для вывода промежуточных и итоговых значений Label (puc. 5). 


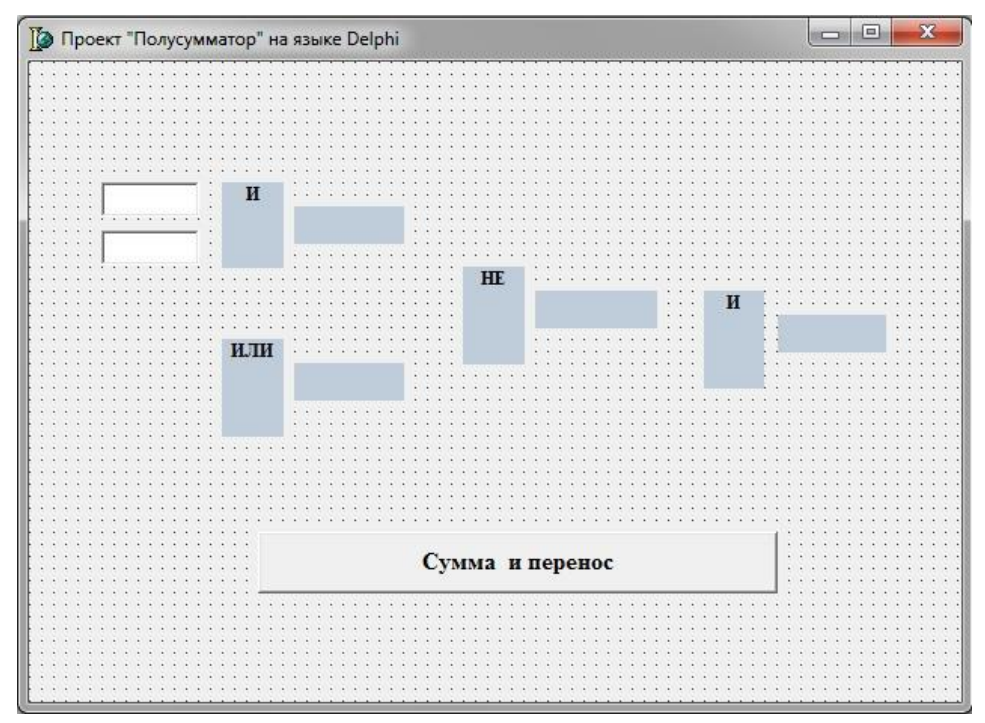

Рис. 5. Интерфейс проекта "Полусумматор"

\section{Разработка событийной процедуры (программного кода):}

Var

//объявить переменнье
A: Boolean;
B: Boolean;
P: Boolean;
S: Boolean;

procedure TForm1.Button1Click(Sender: TObject);

begin

//ввести в текстовые окна начальные значения

$\mathrm{A}:=$ StrToBool(Edit1.Text);

$\mathrm{B}:=$ StrToBool(Edit2.Text);

$\mathrm{P}:=(\mathrm{A}$ And $\mathrm{B})$;

//найти значение переменной $P$

$\mathrm{S}:=(\mathrm{A}$ Or B) And $(\operatorname{Not}(\mathrm{A}$ And B)); $\quad$ //найти значение переменной $S$

//вывести в метки полученные результаты

Label5.Caption:= BoolToStr(P,True);

Label6.Caption:= BoolToStr((A Or B),True);

Label7.Caption:= BoolToStr(Not(P),True);

Label8.Caption:= BoolToStr(S,True); 
end;

end. [4]

\section{Компьютерный эксперимент}

Запустить проект, в текстовые окна ввести логические значения аргументов и щелкнуть по кнопке Сумма и перенос. [4]

\section{Анализ полученных результатов}

На надписи будут выведены логические значения на выходах логических элементов (рис. 6). [4]

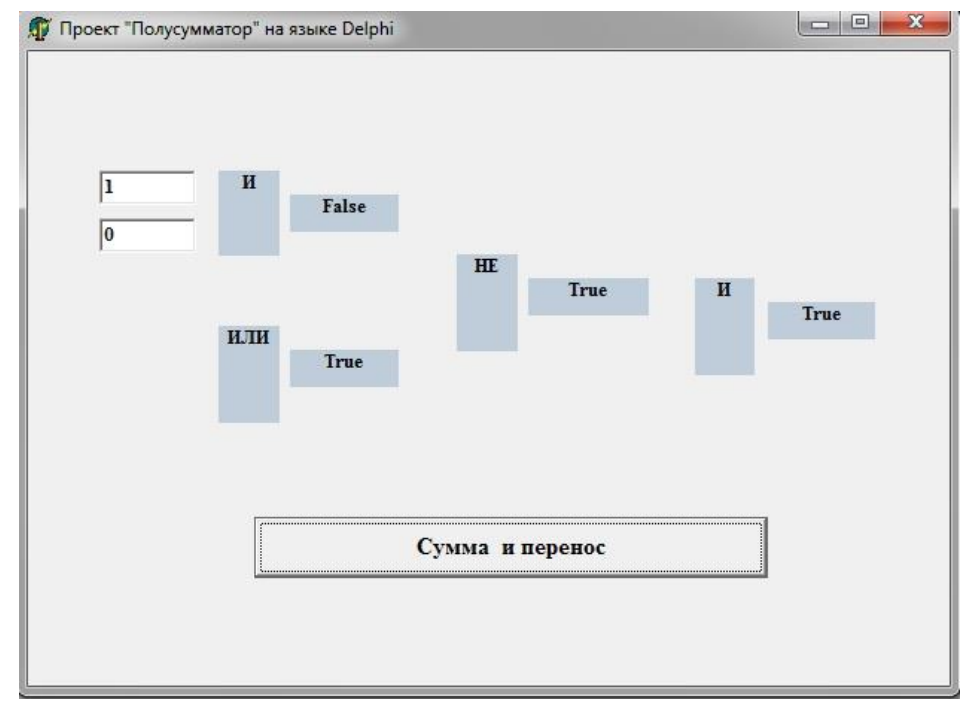

Рис. 6. Выполнение проекта "Полусумматор" в среде Delphi

\section{Заключение}

В скором времени устройства виртуальной реальности станут так же популярныл и функциональны, как мобильные телефоны.

С помощью таких девайсов пользователи смогут смотреть кино и сериалы, присутствовать на массовых мероприятиях и совершать покупки.

Аналитики выяснили, что программы виртуальной и дополненной реальности можно применять в разных сферах деятельности.

При изучении темы “Виртуальная реальность" большую роль для учащихся на практических занятиях имеет разработка проектов для реализации моделирования различных процессов с тестированием технических характеристик. 


\section{Программное обеспечение}

1. Операционная система Windows

\section{2. Система программирования для разработки проектов Delphi}

3. Текстовый процессор MS Word

\section{Список литературы}

1. 9 сфер применения виртуальной реальности [Электронный ресурс]. - Режим доступа: https://vc.ru/flood/13837-vr-use.

2. VR в медицине: современные достижения и перспективы [Электронный ресурс]. - Режим доступа: https://blog.mednote.life/articles/technology/vr-v-medicine.

3. Дополненная реальность в военно-промыщленном комплексе [Электронный ресурс]. - Режим доступа: https://robo-hunter.com/news/dopolnennaya-realnost-v-voenno-promishlennom-komplekse.

4. Исследование информационных моделей. Элективный курс: Учебное пособие / Н.Д. Угринович М.:БИНОМ. Лаборатория знаний, 2004.

5. Краткая история развития технологии виртуальной реальности [Электронный ресурс]. - Режим доступа: https://www.avclub.pro/articles/3d-tekhnologii/kratkaya-istoriya-razvitiya-tekhnologii-virtualnoy-realnosti/.

6. Применение VR/AR-инструментов в искусстве и культуре [Электронный ресурс]. - Режим доступа: https://ar-conf.ru/ru/news/primenenie-vrar-instrumentov-v-iskusstve-i-kulture-59079.

7. Чем виртуальная реальность отличается от дополненной [Электронный ресурс]. - Режим доступа: https://thequestion.ru/questions/317294/chem_virtualnaia_realnost_otlichaetsia_ot_6b95e58b.

8. Что такое виртуальная реальность [Электронный ресурс]. - Режим доступа: https://tproger.ru/translations/vrexplained/. 